\title{
Interactions of numerical and temporal stimulus characteristics on the control of response location by brief flashes of light
}

\author{
J. Gregor Fetterman • P. Richard Killeen
}

Published online: 26 January 2011

(C) Psychonomic Society, Inc. 2011

\begin{abstract}
Pigeons pecked on three keys, responses to one of which could be reinforced after 3 flashes of the houselight, to a second key after 6 , and to a third key after 12 . The flashes were arranged according to variable-interval schedules. Response allocation among the keys was a function of the number of flashes. When flashes were omitted, transitions occurred very late. Increasing flash duration produced a leftward shift in the transitions along a number axis. Increasing reinforcement probability produced a leftward shift, and decreasing reinforcement probability produced a rightward shift. Intermixing different flash rates within sessions separated allocations: Faster flash rates shifted the functions sooner in real time, but later in terms of flash count, and conversely for slower flash rates. A model of control by fading memories of number and time was proposed.
\end{abstract}

Keywords Timing · Counting · Pigeon · Light flashes · Summation

Nonhuman animals can count, but, with a few notable exceptions (e.g., Brannon \& Terrace, 2000), not much or well (Davis \& Pérusse, 1988; Nickerson, 2009; Uttal, 2008). Their ability to discriminate different numerosities, however, can be comparable to that of humans: Pigeons and rats can readily discriminate the number of dots on a screen (Honig \&

J. G. Fetterman $(\bowtie)$

Department of Psychology, Indiana University Purdue University, Indianapolis,

402 N. Blackford St,

Indianapolis, IN 46202, USA

e-mail: gfetter@iupui.edu

P. R. Killeen

Arizona State University,

Tempe, AZ, USA
Stewart, 1989), the number of tones (Davis \& Albert, 1986) and light flashes in a sequence (W. A. Roberts, Coughlin \& Roberts, 2000; W. A. Roberts \& Mitchell, 1994; W. A. Roberts, Roberts \& Kit, 2002), and even the number of responses they have emitted (Fetterman, 1993; Rilling, 1967).

A seminal series of experiments on the similarities of counting and timing by Meck and Church (1983) informed hundreds of subsequent experiments. They demonstrated that rats could time or count auditory signals with equal accuracy, that the performances had similar psychophysical properties and generalized equally well to the tactile modality, that they were similarly affected by drugs, and that the unit of counting was equal to about $200 \mathrm{~ms}$. These findings have been replicated and extended (Meck, Church \& Gibbon, 1985), especially in the elegant research of William Roberts and associates.

Roberts and colleagues' research (e.g., S. Roberts, 1981; W. A. Roberts et al., 2000; W. A. Roberts \& Mitchell, 1994) is central to the experiments presented here. In a series of articles, they studied pigeons' ability to count or to time in the same context, using the peak procedure; these different processes were brought under stimulus control by signaling whether the reinforcer on a given trial was based on the amount of time that had elapsed or the number of stimuli (typically, changes in the color of the key light) that had occurred. The operative contingencies were signaled by the color of the center key light. The main findings were that peak response rates occurred at the programmed time of food reward on timing trials and near the number of light flashes on counting trials. Support for the counting hypothesis was also assayed by varying the rate at which light flashes were delivered from the baseline (intermediate) rate. Response rate curves came to asymptote sooner for the fast flash rate and later for the slow flash rate, as compared with the baseline. The present research addresses the same 
question of how animals parse stimuli that may offer redundant information.

In this article, we use a technique called categorical scaling (Fetterman \& Killeen, 1995) to study pigeons' ability to trisect a stream of flashes of light into the categories few, many, and most. The procedure involves teaching pigeons to peck on three keys, with reinforcement for pecking responses based on the number of light flashes produced by the keypecks on a variable-interval (VI) schedule. Responses to key 1 are reinforced after 3 flashes, to key 2 after 6 flashes, and to key 3 after 12 flashes. Only one of the keys is primed for reinforcement on each trial. The subjects are trained to move from one key to the next on the basis of the number of flashes that occur. We used the categorical-scaling technique to study how pigeons negotiate such contingencies. The research was intended as a parametric exploration of the following points but forced us to a more interesting theoretical hypothesis, presented in the final section of the article. After baseline had been measured, the following procedure was followed.

- The light flashes were removed during a test condition to assess whether the light flashes controlled the pigeons' choices of pecking location. The underlying VI schedule continued to operate in this condition, and reinforcers continued to be delivered after the appropriate number of setups of the schedule, but there were no flashes. We used this manipulation because the birds could have used cumulative time or number of keypecks as the basis of their response decisions, even though neither of these dimensions was as predictive of reinforcement as was flash number.

- Next, we changed the duration of the flashes from $0.3 \mathrm{~s}$ (baseline) to $1.0 \mathrm{~s}$ (test) and then returned to baseline. This manipulation was used to assess whether the birds were summing the accumulated duration of the flashes over time and were using the accumulated total as a cue to choice. If this were the case, longer flashes should affect performance the same way as more light flashes.

- Next, we varied the proportion of trials that ended in reinforcement between 1.0 (every trial ended in reward) and .50 (one half of the trials ended in food reward). These changes were inspired by the theory of timing proposed by Killeen and Fetterman (1988), who predicted that changes in rate of reinforcement would produce changes in pacemaker speed, producing consequent shifts in timing functions. They confirmed these predictions using a timing task (Fetterman \& Killeen, 1995) and a task that required pigeons to count the number of pecks emitted (Fetterman \& Killeen, 2010). If the passage of time is a controlling variable and the above theory is correct, decreasing rate of reinforcement should shift the points of transition to the right. If, however, the number of flashes is the primary controlling variable, there should be little effect on behavior.

- Finally, we varied the rate at which flashes were delivered by manipulating the underlying VI schedule. During training, the schedule was VI 3.5-s; in another condition, three VI schedules were used, with the two new schedules delivering flashes at rates faster or slower than the training schedule. To the extent that real time is a controlling variable, when behavior is plotted as a function of number of flashes, points of transition should shift to the left when flash rate is slowed, since fewer flashes will have occurred by the animals' criterion time to switch. Conversely, when flash rate is sped, more flashes will have occurred by the animals' criterion time, and the functions will shift to the right on a number-of-flashes axis. To the extent that the contingent variable, number of flashes, is a controlling variable, there should be no shift in the functions.

\section{Method}

Subjects

Four adult male Silver King pigeons (Columba livia) maintained at $85 \%$ of their free-feeding weights served as subjects. The pigeons had free access to water and grit and were housed individually in a room with a 12:12-h day: night cycle with dawn at 7 a.m. The pigeons were experimentally naïve at the beginning of the experiment. Key pecking was established through an autoshaping procedure by illuminating each of three pecking keys 30 times per session (total of 90 trials per session), followed by 3 -s access to grain. All the birds reliably pecked all keys after three sessions of autoshaping.

\section{Apparatus}

The experimental enclosure was a standard BRS-LVE three-key operant chamber $(32 \mathrm{~cm}$ high $\times 34 \mathrm{~cm}$ wide $\times$ $34 \mathrm{~cm}$ deep). The pecking keys were accessible through 2$\mathrm{cm}$ circular openings in the work panel on the front wall, with the center of the openings $6.3 \mathrm{~cm}$ apart, $25 \mathrm{~cm}$ above the chamber floor. A force of approximately $0.15 \mathrm{~N}$ was required to operate the keys. The feeder opening was located directly below the center response key and measured $5 \mathrm{~cm}$ on all dimensions; the bottom of the feeder opening was $10 \mathrm{~cm}$ above the chamber floor. When activated, the food hopper provided $3 \mathrm{~s}$ of access to mixed grain. White noise served to mask extraneous sounds; an exhaust fan attached to the chamber wall provided 
additional masking and ventilation. Experimental events were scheduled and recorded by an IBM-compatible PC.

\section{Procedure}

Pretraining Trials began with the illumination of the right key with red light; a single peck to the key produced 3-s access to mixed grain and turned off the light and illuminated the center key with red light. A peck to this key produced mixed grain, turned off the light, and illuminated the left key with red light, a peck to which was followed by reinforcement, and the trial ended. A 15-s intertrial interval (ITI), during which all the lights were off, was followed by the next trial. There were 90 trials per session in this phase of pretraining, which lasted three sessions.

In the next phase of pretraining, keypecks occasionally produced brief (0.3-s) flashes of the houselight. Houselight flashes were produced on a VI schedule with a mean interflash interval (IFI) of $3.5 \mathrm{~s}$. Pecks on the right key produced food after 3 flashes, pecks on the center key produced food after 6 flashes, and pecks on the left key produced food after 12 flashes. Only one key was lit on these pretraining trials, and each key was lit equally often. Finally, as a preliminary to exposing the pigeons to the regular task structure, two kinds of trials were presented, with two keys (right and center or center and left) lit on each trial. Reinforcement was primed on just one of the keys. For instance, if the trial involved illumination of the right and center keys and food was primed on the center key, a pigeon had to switch to the center key before 6 flashes occurred; otherwise, food was not delivered. Similarly, if food was primed on the right key and the pigeon switched to the center key after fewer than 3 flashes, food was not delivered

Baseline After pretraining, trials began with red illumination of all of the keys, with food primed for just one of them; if food for the designated key was missed, the trial immediately ended without reinforcement, and a 15 -s ITI, with all lights in the chamber off, was initiated. As above, pecks to the keys produced light flashes arranged according to a VI 3.5-s schedule, and pecks to the right, center, and left keys could produce reinforcement after 3, 6, and 12 flashes, respectively. Each flash contributed to the cumulative total for the trial. A switch from one key to the next turned off the light on the key that was left; "illegal" switches from key 1 to key 3 terminated the trial. After a week of baseline training, very few illegal switches occurred. All the trials were noncorrection: Success or failure in obtaining reinforcement on one trial had no effect on the requirements for the next trial. By the end of training, the birds missed very few reinforcers (typically $<3$ in a session of 90 trials). The pigeons received 30 sessions of training with the reward on the respective keys associated with 3 (right key), 6 (center key), and 12 (left key) flashes. Once the pigeons' behavior with regard to keypeck location was under control of the number of flashes, a series of manipulations was carried out. These are described below.

Condition 1: Baseline Described above.

Condition 2: Removing the flashes During this session, there were no light flashes, although the underlying VI schedule continued to operate, and pecking at the keys was required to advance the trial. The pigeons could receive reinforcement after 3, 6, or 12 setups of the schedule on the respective keys, but without number of flashes as a cue to the correct peck location. The no-flash condition lasted for 1 session, and then the pigeons were returned to baseline (above) for 10 sessions.

Condition 3: Varying flash duration During training, flashes lasted $0.3 \mathrm{~s}$. The flash duration was changed to $1.0 \mathrm{~s}$, and this change was in effect for 10 sessions, followed by a return to $0.3 \mathrm{~s}$, as in the baseline condition, for 10 sessions.

Condition 4: Changing reinforcer density During training, every correct response was reinforced (100\% payoff). In a subsequent series of conditions, the probability of reinforcement was reduced from $100 \%$ to $50 \%$ for 15 sessions, and then the $100 \%$ condition was reinstated. Trials that did not end with reinforcement were terminated after 12 flashes. The rationale for this manipulation follows that of Fetterman and Killeen $(1995,2010)$; to the extent that the passage of time contributes to the animals' decision to switch, we would expect results similar to the ones that they found: The psychometric functions should be shifted right on a time axis by the reduction in pacemaker speed caused by the reduction in rate of reinforcement and should be shifted to the left when the payoffs are returned to $100 \%$. Each condition lasted 10 sessions; the return to $100 \%$ payoff constituted a return to baseline.

Condition 5: Changing the interflash interval In a final set of conditions, we tested the pigeons with different IFIs. During training the mean IFI was $3.5 \mathrm{~s}$. In another condition, the trained flash rate was intermixed with flash rates that were slower (IFI=5.3 s-"slow) or faster (IFI= $2.4 \mathrm{~s}$ - "fast") than the trained rate. These were chosen to yield a ratio of fast to intermediate and intermediate to slow flash rates of approximately 1.5 . All sets of IFIs contained 12 intervals. This condition was in effect for 10 sessions, and each session contained equal numbers of trials with each flash rate. 


\section{Results}

Baseline Figure 1 shows the main result in terms of peck location based on the number of flashes presented. The figure shows the proportion of total pecks to each key after 3 flashes (right key, unfilled circles), 6 flashes (center key, filled circles), and 12 flashes (left key, squares), and the proportions are based on the last three sessions of the first exposure to baseline conditions. The figure shows that the pigeons were attuned as to where to respond on the basis of the number of flashes that had occurred. The proportion of responses to the right key decreased as the number of flashes increased, with the midpoint of that function at 3 flashes, the reinforced number. The proportion of responses to the center key increased and then decreased, with the peak of that function around 6 flashes; the proportion of responses to the left key showed a monotonic increase, with the function peaking after 12 flashes had occurred. We conclude (see below for supporting evidence) that the pigeons decided where to peck on the basis of the accumulated memory of light flashes.

Figure 2 shows data similar to those presented in Fig. 1, except that the choice proportions are plotted as a function of time rather than number of flashes. As in Fig. 1, the data were taken from the last three sessions of initial baseline testing. Although the houselight flashes were presented on a variable schedule, there was still a correlation (around .7) between flash number and time elapsed. The birds could have used time as the primary cue about where to peck to receive reinforcement; this figure indicates that the timing hypothesis is plausible. Choices among the three keys show the same pattern as that displayed in Fig. 1:a decreasing allocation to key 1 , an increasing then decreasing allocation to key 2, and an increasing allocation to key 3 .

Figures 1 and 2 show relative rates on each of the keys as a function of number (Fig. 1) and time (Fig. 2). Hereafter, we report relative rates on the center (6 flashes) key alone, $p_{6}$. This method of presentation is economical and contains virtually all of the information provided by plots of responding on all three keys. The left limb of the center key function is approximately the inverse of the " 3 " key function $\left(1-p_{3}\right)$, and the right limb of the center key function is approximately the inverse of the "12" key function ( $-p_{12}$; see Figs. 1 and 2$)$. Hence, there is very little loss of information in this focus of attention on the center key performance.

The median numbers of flashes at the points of transition were 3.2 and 8.1, with median standard deviations of 1.52 and 2.25. If counting showed scalar variability, we would be able to predict this last number as $1.52(8.2 / 3.1)$. That prediction, 4.0 , is in gross error. If counting showed Poisson variability, as might be the case with occasional dropped or added counts (Killeen, 2002), we would predict it as $1.52(8.2 / 3.1)^{1 / 2}$. That prediction, 2.4, is closer to the mark, suggesting that counting error here is Poisson. However, by adding an intercept parameter, the generalized Weber fraction could, of course, account for these data perfectly.
Fig. 1 Proportion of responses to the few (3 flashes), many (6 flashes), and most (12 flashes) keys as a function of number of flashes
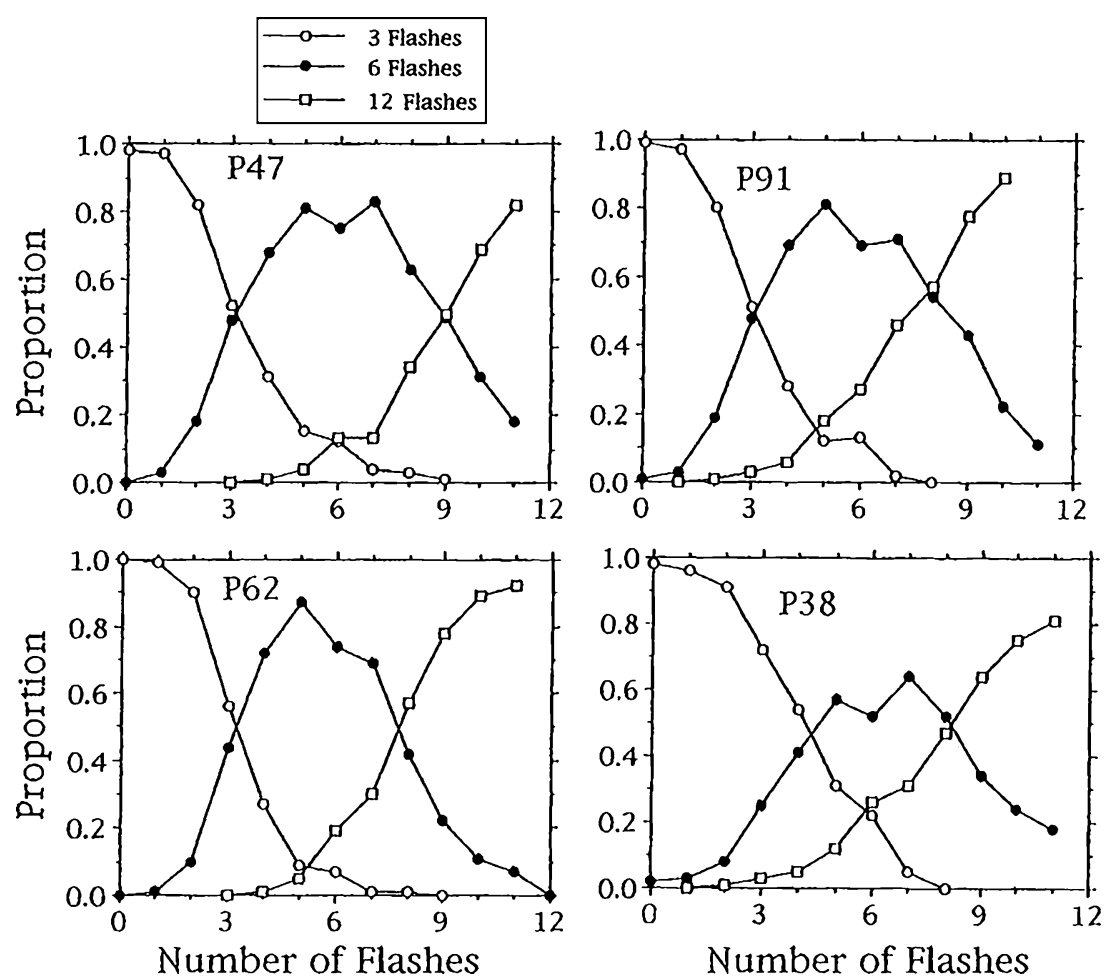
Fig. 2 Proportion of responses to the few (3 flashes), many (6 flashes), and most (12 flashes) keys as a function of time, segmented into 2-s bins

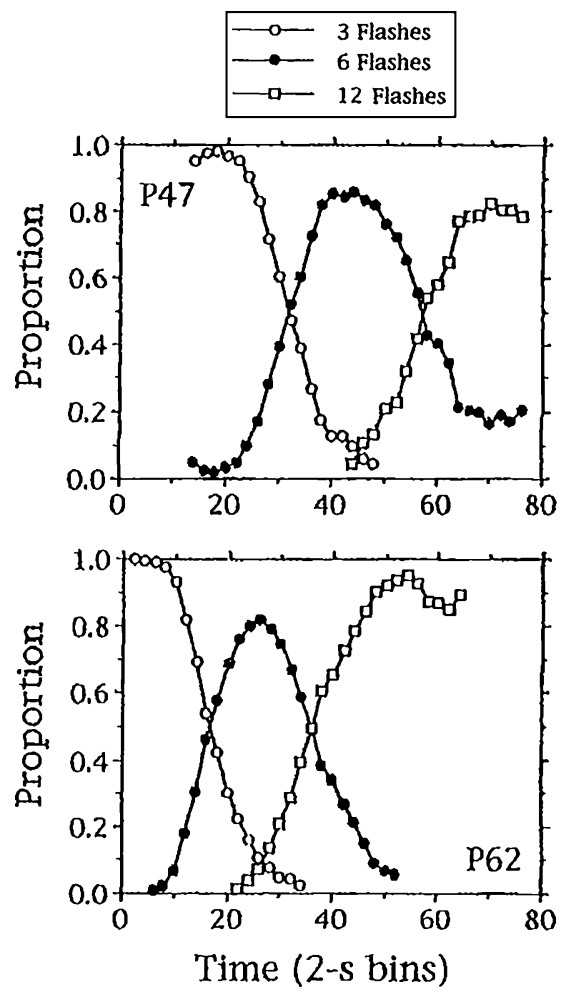

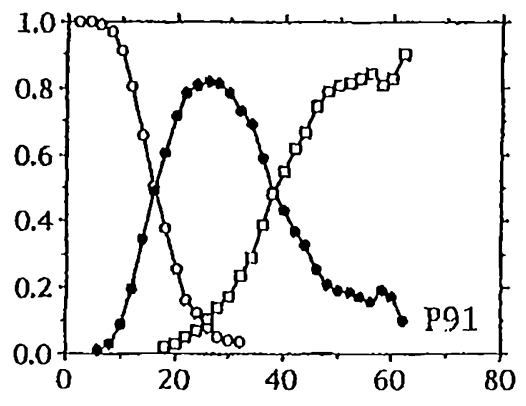

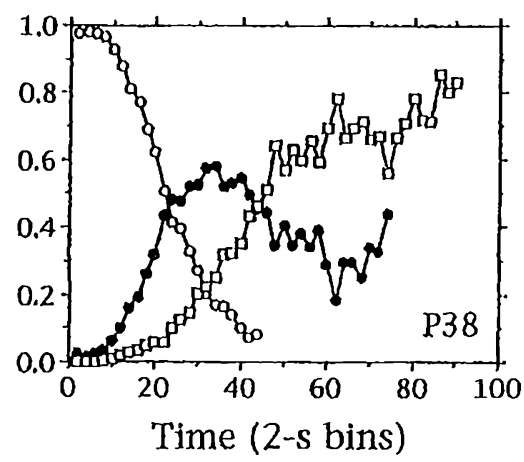

Condition 2 Figure 3 shows the data from the single test session in which the flashes were removed. The data are shown as the proportion of responses to the center key as a function of time through the trial. Data are shown for the session before flashes were removed and for the session without flashes. Removing the flashes severely disrupted performance: It delayed changes in and out of the center key past their optima for P47 and completely erased the pattern for the other 3 birds. Instead of the modal time of center key responding at 25-30 s, their key transitions radically slowed, with the time of maximal responding on the center key exceeding $50 \mathrm{~s}$. Whereas the ambivalent results of the baseline condition with respect to the locus of control could be attributed to the confounding of progression of time with flash number, the present condition shows that control by flash number was predominant in 3 of 4 birds and played some role, possibly additive, for P47. Either of two hypothetical mechanisms-the necessity of flashes for transition, so that their absence stalled the pigeons, or generalization decrement-could be adduced to account for these data. But the latter might also predict random pecking, rather than the slow transitions seen.

Condition 3 Figure 4 shows the effect of changing the duration of the houselight flashes from 0.3 to $1.0 \mathrm{~s}$, using data from the last three sessions of each flash duration condition. The dependent measure is the proportion of responses to the center " 6 " key. Three pigeons displayed a leftward shift when flash duration was increased; pigeon P38 did not behave differently when flash duration was changed. For the other pigeons, longer cue lights prompted earlier switching. If the birds were simply counting the number of flashes, changing their duration should not have shifted any of the curves. Longer flashes seem to have a greater impact on the count than do smaller ones. It was noted in the introduction that Meck and Church (1983) found that $200 \mathrm{~ms}$ of signal was equivalent to a single count for rats. Might something similar be going on here? If extended stimuli are tantamount to multiple counts, this could account for the shift, where it occurred. If so, then counting means timing the cumulative duration of the stimulus. Condition 5 tested this hypothesis.

Condition 4 Figures 5 and 6 show the effects of changing the probability that a trial would end in reinforcement. All the analyses used data from the last three sessions of each probability condition. Unfilled circles represent performance before the change, and filled circles represent performance after the change. As above, the dependent measure is the proportion of responses to the center key as a function of the number of flashes. When reinforcement probability was decreased, all the birds displayed a shift to the right, after more flashes. Pigeon P91 showed the largest effect of the change, and pigeon P38 once again the smallest. When reinforcement probability was increased (Fig. 6), 3 of the pigeons switched after fewer flashes. The 
Fig. 3 Proportion of responses to the center (6 flashes) key as a function of time. Data are shown for the baseline condition, where pecks produced flashes on a variable interval (VI) schedule (unfilled circles). The filled circles show data from a condition without flashes, wherein pecking served only to advance the underlying VI schedule
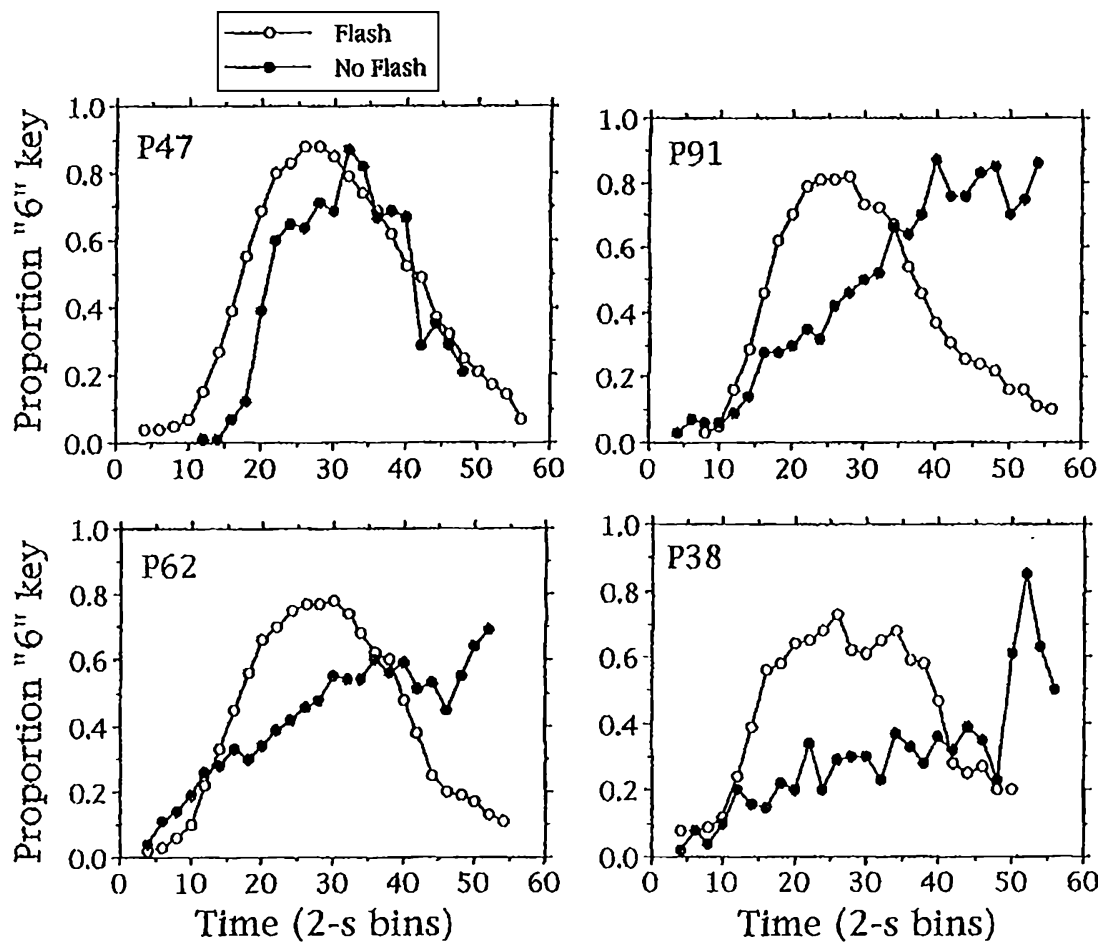

exception was P38, who showed no change in the first point of transition but made the transition to the last key sooner at the higher reinforcement probability.

If the animals were simply counting, changing the probability of reinforcement should not have had these systematic effects; if they were timing the duration of the flashes and their clock ran faster under the higher rate of reinforcement, the higher rate of reinforcement should lend the semblance of more stimulation, and they would shift sooner, as they largely did; and they should shift later under the reduced probability of reinforcement. The fifth condition shows, however, that this cannot be a complete explanation.

If the animals' behavior were under partial control by real time and their clock ran slower under lowered rate of reinforcement, they would shift later, in terms of both number of flashes (Fig. 5) and number of seconds. The converse holds for increased rate of reinforcement (Fig. 6).
Fig. 4 Proportion of responses to the center (6 flashes) key as a function of the number of flashes. Data are shown for the trained value of 0.3 -s light flashes (unfilled circles) and the condition in which the flash duration was increased to $1.0 \mathrm{~s}$ (filled circles)
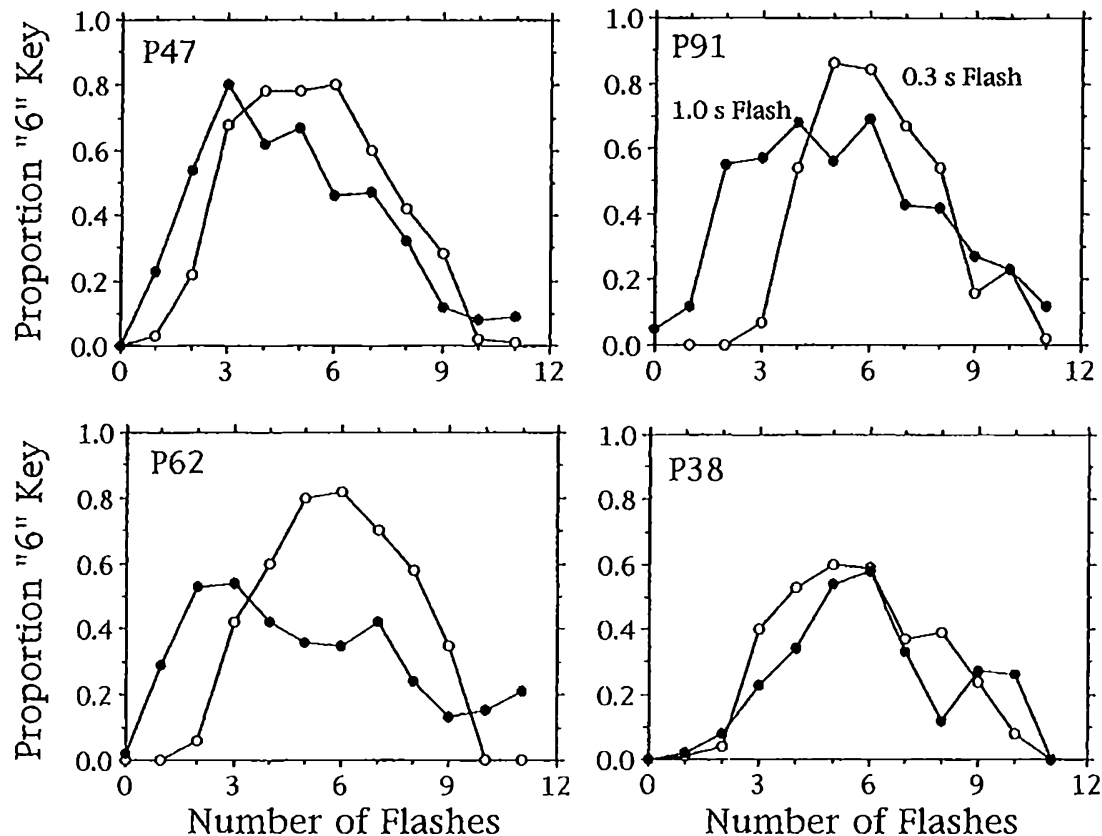
Fig. 5 Proportion of responses to the center " 6 " key as a function of the number of flashes. Data are from the first condition, in which every trial ended with reinforcement $(1.0$; unfilled circles), and the second condition, in which $50 \%$ of the trials ended with reinforcement (.50; filled circles)
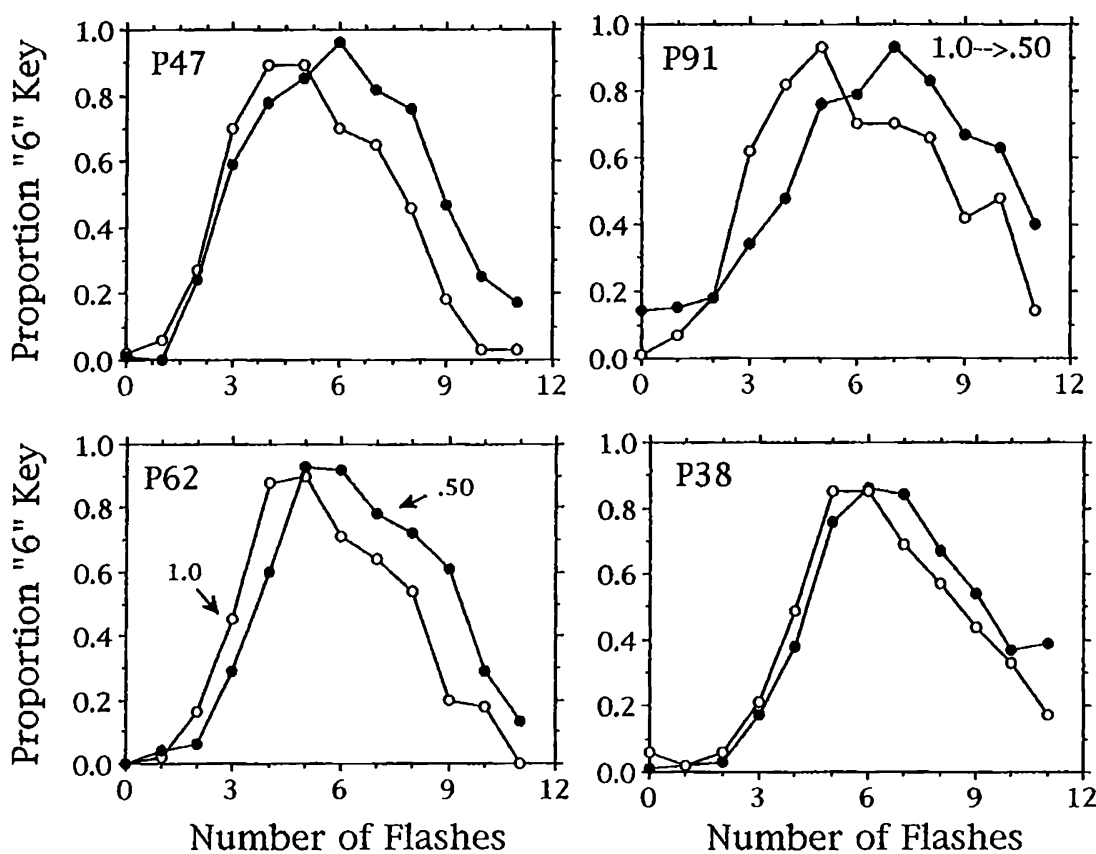

Condition 5 In a final set of conditions, we varied the rate at which flashes were produced by pecks to the keys; the baseline was VI 3.5-s, and the faster and slower flash rates had periods of 2.4 and $5.3 \mathrm{~s}$, respectively. When variable flash rates were used, the correlation between number of flashes and the time taken to produce the flashes was attenuated, as shown in Fig. 7, which gives the correlations between the number of flashes produced before a switch from key 1 to key 2 and the time taken before the switch; the corresponding measures are shown for switches from key 2 to key 3 . The correlations are based on data pooled across all subjects. The figure demonstrates that the correlations between time and number were reduced when variable flash rates were used, as compared with the fixed flash rate condition.

Figure 8 shows the center key rate functions for each of the flash rates (intermediate baseline, fast, slow). The trend was that the allocation of responses for the fast rate was displaced to the right (more flashes to transit), as compared with baseline, and that for the slow rate, the allocation was shifted to the left. All the birds showed this effect, although the influence of flash rate was greater for some birds than for others. If the birds were simply counting, there should have been no shift; if they were timing or using a combination of counting and timing, the shift occurred in the predicted direction. The difference in flash rates between the fast and slow conditions is greater than $2 / 1$; if the pigeons were purely timing, the number of flashes before switching should be more than twice as great at the fast flash interval as at the slow, which was not the case.

The upper panels of Fig. 9 show the mean number of flashes that occurred before a switch from key 1 to key 2 (upper left panel) and the corresponding measure for switches from key 2 to key 3 (upper right panel). The regression lines would be flat if the pigeons were purely counting, but the slopes are negative, indicating that the pigeons produced fewer flashes before a switch as the IFI was lengthened. Mean slopes were -0.31 and -0.75 for data in the upper left and right panels, respectively.

The corresponding switch measures based on the time before a switch are shown in the bottom panels of Fig. 9. If the pigeons timed their switches, the regression lines would be horizontal. But the regressions have a positive slope, indicating that the time to switch increased as the IFI lengthened. Mean slopes were 3.15 and 4.56 for data in the lower left and right panels, respectively. In toto, Figs. 8 and 9 indicate that both the number of flashes and the time taken to produce the flashes controlled the birds' choices.

\section{Discussion}

Synopsis and problematics Pigeons learned to move across three keys on the basis of the number of light flashes produced by their pecks according to a VI 3.5-s schedule. Pecks to one key were rewarded after 3 flashes, to a second key after 6 flashes, and to a third key after 12 flashes. The pigeons' behavior was apparently controlled by the number of flashes produced. They pecked on the first key until 3 or more flashes occurred; absent reinforcement, they soon switched to the second key, pecking and producing flashes. If food was not obtained after 6 flashes, at some point 
Fig. 6 Proportion of responses to the center key as a function of the number of flashes. Data are shown from the first condition, in which $50 \%$ of the trials ended with reinforcement $(.50$; unfilled circles), and from the second condition, in which every trial ended with reinforcement $(1.0$; filled circles)
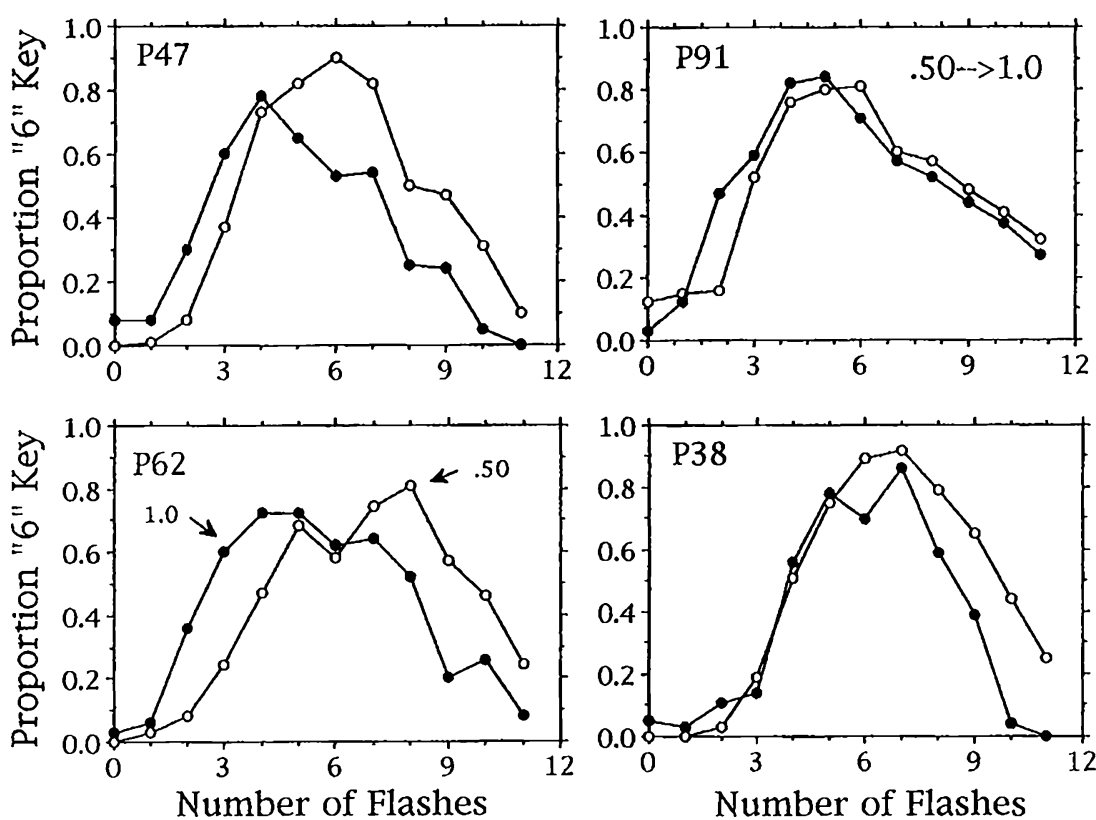

thereafter, averaging 8 flashes, they moved to the third key and continued to peck, producing flashes, until food was obtained or the trial ended without reinforcement.

Initial analyses with time as the predictor (Fig. 2) showed that the pigeons could have based decisions about when to switch between keys on the passage of time, rather than on the number of flashes that had occurred (Fig. 1); the correlations between these two measures was about .70. But performance deteriorated when the flashes were removed (Fig. 3), showing that the flashes were key discriminative cues for making transitions between the keys.

Condition 3 showed that the duration of the flashes made an important difference in performance (Fig. 4). All the birds were trained with flashes that lasted $0.3 \mathrm{~s}$. In a subsequent

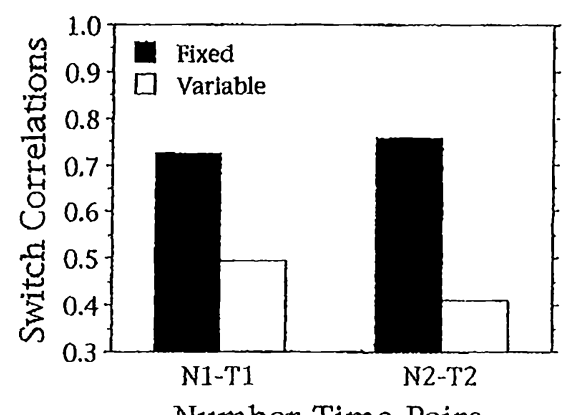

Fig. 7 Correlations between the number of flashes before a switch from key 1 to key 2 and the time of the switch, and similar correlations for switches from key 2 to key 3. Data are shown for a condition in which the underlying variable interval (VI) schedule ( $3.5 \mathrm{~s})$ was the same across trials (fixed; filled bars). The same data are shown for a condition in which three VI schedules were intermixed within sessions (VI 2.4, VI 3.5, VI 5.3; variable; unfilled bars) condition, the flash duration was changed to $1.0 \mathrm{~s}$ and then returned to $0.3 \mathrm{~s}$. The increase in flash duration produced a leftward shift in the choice functions. It is as though the longer flash durations produced an increase in the cumulative number of counts, as compared with the 0.3 -s condition; but simple concepts of counting do not allow this effect.

In Condition 4, the probability of reinforcement was varied, from 1.0 to .5 and then from .5 to 1.0 . When the probability was decreased (Fig. 5), the curves shifted to the right, as compared with the baseline. When the probability was increased (Fig. 6), the curves shifted to the left. These manipulations were motivated by the behavioral theory of timing (Killeen \& Fetterman, 1988), in which the speed of a pacemaker varies with the rate of reinforcement (Killeen, Bizo \& Hall, 1999). If the animals were timing and their pacemaker were slowed by decreasing rates of reinforcement, the allocation of responses would shift to the right on a realtime axis: It would take them longer to get to their criterial times. Since they would be switching later in real time, more flashes would have occurred. Thus, on these axes, a decrease in rate of reinforcement that slowed a pacemaker should cause a rightward shift. It did. If the animals were solely counting flashes, they would switch at the same number of flashes, and the curves superimpose. They did not.

In the fifth condition, the rate of flashes varied within sessions. During training, flashes were produced according to a VI 3.5-s schedule; in the mixed condition, different flash rates were presented within each session. When the flashes came quickly, the pigeons waited for a larger number to shift; when they came slowly, they shifted after fewer (Figs. 8 and 9). Again, singular accounts of these effects are nonobvious. If their behavior were predicated on 
counting alone, there should be no effect on a number of flashes axis (Fig. 8); if it were predicated on timing alone, the regressions in the bottom panels of Fig. 9 should have slopes of 0 ; they had positive slopes.

A hypothetical mechanism Recent work by W. A. Roberts (2010) helps to demystify the present results. Roberts showed that pigeons quickly forget prior stimuli. His theoretical decay of memory function was quite similar to the empirical ones of Killeen (2001). Consider a model in which memory for flashes grows exponentially with their duration - in particular, as a concave cumulative exponential function. Assume also that that memory decays exponentially with the time since their offset. An instance of such a model is an exponentially weighted moving average of the time the light is on (Killeen, 1981). The growth of memory for houselight on will then be a sawtooth concave increasing function, much like the curves shown in the bottom panels of Roberts et al.'s (2002) Figs. 1, 2 and 3. Letting $m$ stand for accumulated memory of the stimulus, when the trial ends with the end of the stimulus, the accumulated memory at the end of the $n$th trial is the residual from the prior trial, decayed by an exponential factor with time constant $c_{\text {off }}$, plus the new memory, accumulated with the time constant $c_{\text {on }}$ :

$m_{n}=\mathrm{e}^{-t_{o f f} / c_{o f f}} m_{n-1}+1-\mathrm{e}^{-t_{o n} / c_{o n}}$.

This hypothesis is essentially identical with one proposed by Keen and Machado to account for their frequency discrimination results (Keen \& Machado, 1999; Machado $\&$ Keen, 2002), the main difference being the detail of their assuming linear accumulation and the present model's assuming concave accumulation. With constant stimulus durations such as those they used, there is no difference on this point. Also, for precision, they included a constant for proactive inhibition, which might be realized in this model by a nonzero value for $m_{0}$. The model has functional similarities to the timing model of Staddon and associates (Staddon, Chelaru \& Higa, 2002; Staddon \& Higa, 1999), in that the durations of both the events timed and the times between events will affect behavior.

If pigeons set a criterion for switching based on the total amount of houselight remembered, the following should occur. (1) When flashes are removed, key transition will be shifted later, to the right, as was shown to be the case in condition 2 in this article. (2) When flashes are made longer, each adds more to the memory, criteria will be met sooner, and transitions will shift to the left, as was shown to be the case in condition 3. (3) When rate of reinforcement is decreased, rate of responding also decreases, causing a decrease in the frequency of the flashes (payoff on these very short VI schedules covaries with rate of responding); it should take longer to accumulate enough flash memory, and transitions should require more flashes and shift to the right, as was shown to be the case in condition 4. (4) When flashes occur more quickly, there is less time for their memorial decay, criterion will be reached sooner, and transitions will occur earlier, which was shown to be the
Fig. 8 Proportion of responses to the center key as a function of the number of flashes. These data are shown for each of the flash rates used in the variable flash rate condition (medium [baseline $]=$ variable interval $(\mathrm{VI})$ $3.5-\mathrm{s}$; fast $=$ VI $2.4-\mathrm{s}$; slow $=\mathrm{VI}$ $5.3-\mathrm{s})$
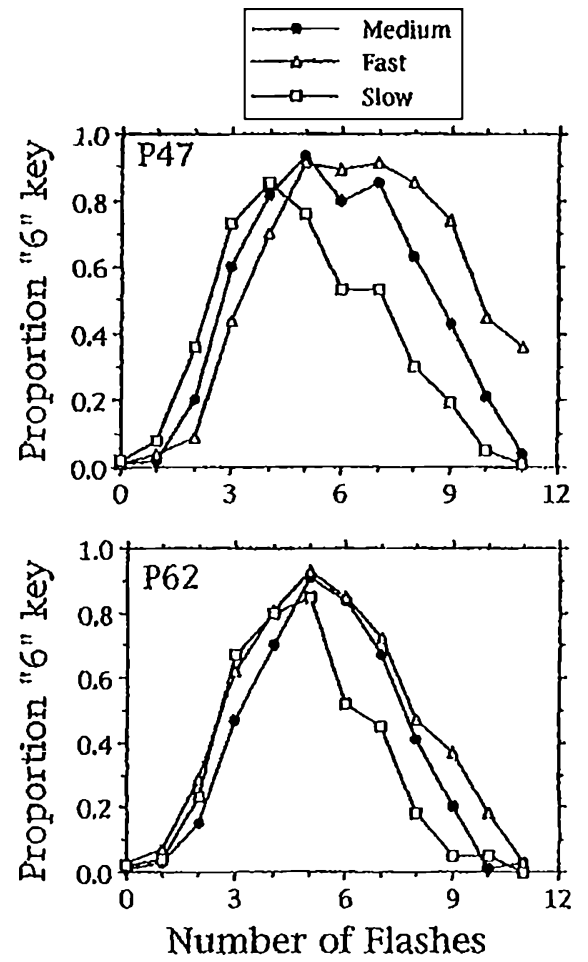

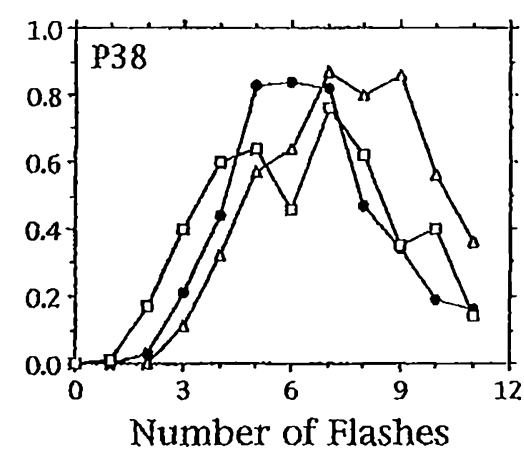


case in condition 5 (bottom panels of Fig. 9). However, transitions should also have occurred after fewer flashes, since each would have added to the next more effectively; but this prediction is disconfirmed by the negative, rather than positive, slope in the top panels of Fig. 9.

Condition 5 falsified Eq. 1 as a complete explanation, because the data shifted in a direction contrary to predictions. Both time and number matter in these experiments. Perhaps this is not surprising, since both are correlated with correct performance. Perhaps a metric of time/events, $M_{n, t}$, comprising a weighted sum of the memory for the events to be counted, $m_{n}$, and real elapsed time $t$ converted to a common dimension, such as number of counts, by any of a variety of theories of timing, should be used:

$M_{n, t}=\alpha m_{n}+(1-\alpha) c t$.

When this metric exceeds a criterion, transitions are made to the next key. Qualitatively, Eqs. 1 and 2 could map all of the data reported here, although they overpower those data and have an ad hoc flavor.

Several testable new predictions follow from Eq. 1. (1) Only flashes at a moderate rate will work: Too infrequent and early flashes will be forgotten by the time of subsequent ones; if they are too frequent, memory will be saturated. (2) There will be an interaction between flash duration and frequency: At high flash rates, brief stimuli will work better than longer ones, and conversely. (3) Some of the variance in points of transition should be attributable to the particular VI intervals that are operative: When they happen to be widely spaced, switching will be delayed; when they are close together, switching will be advanced. (4) Shifting the signal presentation to a ratio schedule should speed response rates and, thus, stimulus delivery, causing transitions to occur sooner.

Several testable implications follow from this hypothesis that are not necessary for the validity of the model but would constitute interesting extensions of it. (1) Probing with brighter stimuli may hasten transitions. (2) Probing while shortening the ITI may cause stimuli from the prior trials to enter the sum (a kind of proactive addition), hastening the transitions. (3) Probing with ITIs during which houselights are on may hasten transitions. (4) Modulating ambient illumination during trials may have a positive correlation with speed of transition.

If the concave accumulation of memory and convex decumulation of it holds for stimuli other than light flashes, we might expect the following. (1) When asked to count pecks, brief time-outs after each response should debase memory for them, shifting transitions to the right. This has been found (Fetterman \& Killeen, 2010). (2) Because the accumulation of memory is concave, animals should perform relatively better when discriminating small numbers of events, rather than larger ones. Such a magnitude effect is well known (e.g., W. A. Roberts, 2010). (3) When attention
Fig. 9 Means of switching from key 1 to key 2 (left column) and from key 2 to key 3 (right column) as a function of interflash interval. Data are shown separately for the mean number of flashes to a switch (top row) and for the mean time to a switch (bottom row). If the pigeons were just counting, the regressions in the top panels should be flat; if they were just timing, the regressions in the bottom panels should be flat
Key 1-->Key 2
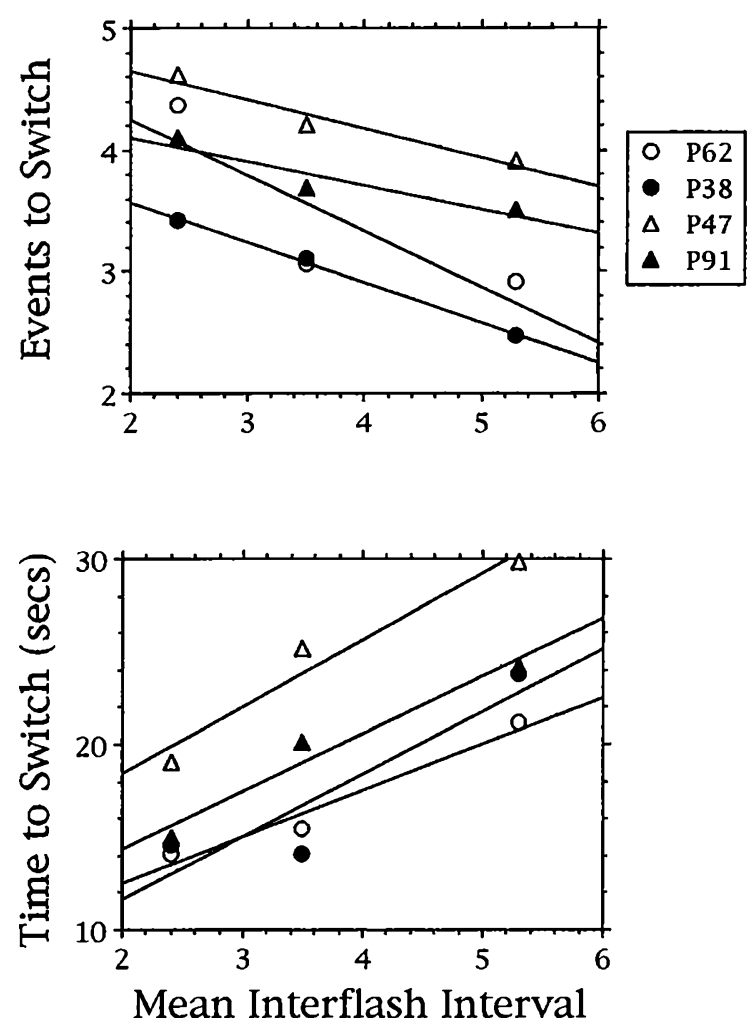

Key 2-->Key 3

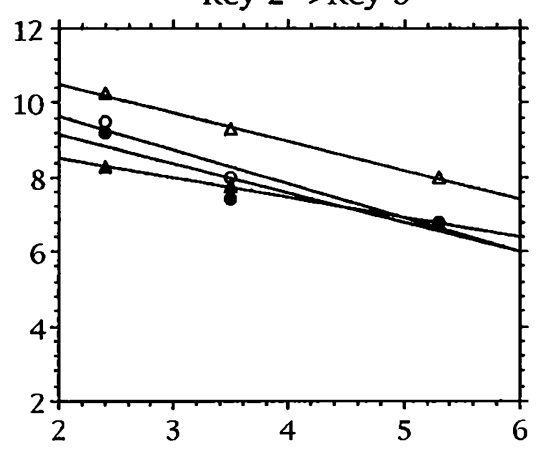


is distracted from stimuli, they should seem shorter. Zentall reported that requiring pigeons to peck during a timing task causes them to shorten their estimates of stimulus duration (Zentall \& Singer, 2008). (4) Ability to discriminate the number of events such as reinforcers may be enhanced by spacing them, so that those extended stimuli do not saturate memory. Zentall has recently reported just such an effect (Rayburn-Reeves, Miller \& Zentall, 2010). (5) When report of a discrimination is delayed, the decaying traces will shift judgments toward the fewer events. This has been validated (Fetterman \& MacEwen, 1989, 2003) and is consistent with the classic choose-short effect for delayed report of temporal extent, also plausibly due to the same mechanism (Killeen \& Fetterman, 1988).

In summary, Roberts and colleagues (e.g., W. A. Roberts \& Boisvert, 1998) have shown that animals can use either time or number to guide their behavior. Pacemaker-timer theories are count-based theories of timing. Keen and Machado's (1999) theory of counting as a moving average of memory for events accounts for some of the present results and for others in the literature and supports strong and testable predictions. Its functional similarity to the multiple trace model of Staddon and associates (Staddon et al., 2002; Staddon \& Higa, 1999) lends hope for a unified account of counting and timing in nonverbal organisms. Perhaps this would be one such as the following. Nonverbal animals base discriminations on their memory for criterial events at the time of the question; in timing experiments, this is an avatar of real time, such as a sequence of adjunctive responses or hypothetical memorial counts; in counting experiments, this is memory for the event to be counted. These different cues may blend, as in Eq. 2, with memories of them increasing as a concave function of exposure and decreasing as a convex function of delay to question.

\section{References}

Brannon, E. M., \& Terrace, H. S. (2000). Representation of the numerosities 1-9 by rhesus macaques (Macaca mulatta). Journal of Experimental Psychology: Animal Behavior Processes, 26, 31-49.

Davis, H., \& Albert, M. (1986). Numerical discrimination by rats using sequential auditory stimuli. Learning \& Behavior, 19, 57-59.

Davis, H., \& Pérusse, R. (1988). Numerical competence in animals. Behavioral and Brain Sciences, 11, 561-579.

Fetterman, J. G. (1993). Numerosity discrimination: Both time and number matter. Journal of Experimental Psychology: Animal Behavior Processes, 19, 149-164.

Fetterman, J. G., \& Killeen, P. R. (1995). Categorical scaling of time: Implications for clock-counter models. Journal of Experimental Psychology: Animal Behavior Processes, 21, 43-63.

Fetterman, J. G., \& Killeen, P. R. (2010). Categorical counting. Behavioural Processes, 85, 28-35.

Fetterman, J. G., \& MacEwen, D. (1989). Short-term memory for responses: The "choose-small" effect. Journal of the Experimental Analysis of Behavior, 52, 311-324.
Fetterman, J. G., \& MacEwen, D. (2003). Acquisition and retention in compound matching with hue and peck number elements. Learning and Motivation, 34, 354-371.

Honig, W. K., \& Stewart, K. E. (1989). Discrimination of relative numerosity by pigeons. Animal Learning \& Behavior, 17, 134146.

Keen, R., \& Machado, A. (1999). How pigeons discriminate the relative frequency of events. Journal of the Experimental Analysis of Behavior, 72, 151-175.

Killeen, P. R. (1981). Averaging theory. In C. M. Bradshaw, E. Szabadi, \& C. F. Lowe (Eds.), Quantification of steady-state operant behaviour (pp. 21-34). Amsterdam: Elsevier.

Killeen, P. R. (2001). Writing and overwriting short-term memory. Psychonomic Bulletin \& Review, 8, 18-43.

Killeen, P. R. (2002). Scalar counters. Learning \& Motivation, 33, 63-87.

Killeen, P. R., Bizo, L. A., \& Hall, S. (1999). A clock not wound runs down. Behavioural Processes, 45, 129-139.

Killeen, P. R., \& Fetterman, J. G. (1988). A behavioral theory of timing. Psychological Review, 95, 274-295.

Machado, A., \& Keen, R. (2002). Relative numerosity discrimination in the pigeon: Further tests of the linear-exponential-ratio model. Behavioural Processes, 57, 131-148.

Meck, W. H., \& Church, R. M. (1983). A mode control model of counting and timing processes. Journal of Experimental Psychology: Animal Behavior Processes, 9, 320-334.

Meck, W. H., Church, R. M., \& Gibbon, J. (1985). Temporal integration in duration and number discrimination. Journal of Experimental Psychology: Animal Behavior Processes, 11, 591-597.

Nickerson, R. S. (2009). Mathematical reasoning: Patterns, problems, conjectures, and proofs. London: Psychology Press.

Rayburn-Reeves, R. M., Miller, H. C., \& Zentall, T. R. (2010). "Counting" by pigeons: Discrimination of the number of biologically relevant sequential events. Learning \& Behavior, $38,169-176$.

Rilling, M. (1967). Number of responses as a stimulus in fixed interval and fixed ratio schedules. Journal of Comparative and Physiological Psychology, 63, 60-65.

Roberts, S. (1981). Isolation of an internal clock. Journal of Experimental Psychology: Animal Behavior Processes, 7, 242-268.

Roberts, W. A. (2010). Distance and magnitude effects in sequential number discrimination by pigeons. Journal of Experimental Psychology: Animal Behavior Processes, 36, 206-216.

Roberts, W. A., \& Boisvert, M. J. (1998). Using the peak procedure to measure timing and counting processes in pigeons. Journal of Experimental Psychology: Animal Behavior Processes, 24, 416430.

Roberts, W. A., Coughlin, R., \& Roberts, S. (2000). Pigeons flexibly time or count on cue. Psychological Science, 11, 218-222.

Roberts, W. A., \& Mitchell, S. (1994). Can a pigeon simultaneously process temporal and numerical information? Journal of Experimental Psychology: Animal Behavior Processes, 20, 66-78.

Roberts, W. A., Roberts, S., \& Kit, K. A. (2002). Pigeons presented with sequences of false flashes use behavior to count but not to time. Journal of Experimental Psychology: Animal Behavior Processes, 28, 137-150.

Staddon, J. E. R., Chelaru, I. M., \& Higa, J. J. (2002). A tuned-trace theory of interval-timing dynamics. Journal of the Experimental Analysis of Behavior, 77, 105-124.

Staddon, J. E. R., \& Higa, J. J. (1999). Time and memory: Towards a pacemaker-free theory of interval timing. Journal of the Experimental Analysis of Behavior, 71, 215-251.

Uttal, W. R. (2008). Time, space, and number in physics and psychology. Cornwall-on-Hudson, NY: Sloan.

Zentall, T. R., \& Singer, R. A. (2008). Required pecking and refraining from pecking alter judgments of time by pigeons. Learning \& Behavior, 36, 55-61. 